\title{
Motor Function of the Opossum Sphincter of Oddi
}

\author{
James Toouli, Wylie J. Dodds, Ryuichi Honda, Sushil Sarna, \\ Walter J. Hogan, Richard A. Komarowski, John H. Linehan, and \\ Ronald C. Arndorfer, Departments of Medicine, Radiology, Pathology, and \\ Surgery, The Medical College of Wisconsin, Milwaukee, Wisconsin 53226
}

\begin{abstract}
A B S TRACT We studied the opossum sphincter of Oddi (SO) because in this species the $\mathrm{SO}$ is $\sim 3 \mathrm{~cm}$ in length and its extraduodenal location permits recording of motor activity with negligible interference from duodenal motor activity. The SO segment of 120 animals was evaluated by one or more of the following: (a) intraluminal manometry; (b) electromyography; (c) common bile duct (CBD) flow monitored by a drop counter; $(d)$ cineradiography of intraductal contrast medium; and (e) histologic examination. SO pullthroughs using an infused catheter of $0.6-\mathrm{mm}$ o.d. invariably showed a high pressure zone (HPZ) of $18 \pm 3$ $\mathrm{SE} \mathrm{mm} \mathrm{Hg}$ in the terminal 4-5 $\mathrm{mm}$ of the SO segment. This HPZ had a narrow lumen, $0.5-0.7 \mathrm{~mm}$ in diam, and prominent circular muscle. The HPZ in the terminal SO had both active and passive components. HPZ with minimal amplitude and a paucity of underlying smooth muscle were present inconstantly at the junction of the SO segment with the CBD and pancreatic duct, respectively. The dominant feature of the SO segment was rhythmic peristaltic contractions that originated in the proximal SO and propagated toward the duodenum. These contractions occurred spontaneously at a rate of $2-8 / \mathrm{min}$, ranged up to $200 \mathrm{~mm} \mathrm{Hg}$ in magnitude, had a duration of $\sim 5$ $s$ and were not abolished by tetrodotoxin. Concurrent myoelectric and manometric recordings showed that each phasic contraction was immediately preceded by an electrical spike burst. Simultaneous recordings of
\end{abstract}

A preliminary communication of this work was presented at the Meeting of the American Motility Society in Tucson, AZ, October, 1980 and appeared in abstract form in 1980 . Dig. Dis. Sci. 25: 719 a. (Abstr.)

During the time this study was performed Dr. Toouli was the recipient of an Applied Health Science Fellowship from the Australian National Health and Medical Research Council. His current address is Department of Surgery, Flinders Medical Centre, Bedford Park, South Australia, 5042, Australia.

Received for publication 4 November 1981 and in revised form 24 June 1982. cineradiography, CBD inflow of contrast medium, SO manometry, and SO electromyography indicated that rhythmic peristaltic contractions stripped contrast medium from the SO into the duodenum. During SO systole, CBD emptying was transiently interrupted, whereas SO filling occurred during the diastolic interval between SO peristaltic contractions. SO distention increased the frequency of SO peristalsis. We conclude that $(a)$ the dominant feature of the opossum SO is rhythmic peristaltic contractions that originate in the proximal SO and propagate toward the duodenum; (b) these forceful SO peristaltic contractions are myogenic in origin and serve as a peristaltic pump that actively empties the SO segment; (c) CBD outflow occurs passively during $\mathrm{SO}$ diastole, but is interrupted transiently during each SO peristaltic contraction; and (d) a short HPZ with active as well as passive components exists in the distal SO segment and acts as a variable resistor to $\mathrm{SO}$ outflow.

\section{INTRODUCTION}

Motor dynamics of the sphincter of Oddi $(\mathrm{SO})^{1}$ remain incompletely understood (1-3). Previous studies have been hampered by the fact that the SO segment is located largely within the duodenal wall in most animal species $(4,5)$. Such anatomy makes it difficult to record SO motor activity without interference from duodenal motor activity. In this study we selected the opossum for investigation because the $\mathrm{SO}$ in this species measures about $3 \mathrm{~cm}$ in length and is largely extraduodenal. Further, the opossum SO exhibits prominent rhythmic phasic contractions (6-8) similar in frequency and pressure amplitude to those recorded in the human SO (9-11). Our major aims were to $(a)$

\footnotetext{
${ }^{1}$ Abbreviations used in this paper: $\mathrm{CBD}$, common bile duct; HPZ, high pressure zone; PD, pancreatic duct; PV, polyvinyl; SBF, spike burst frequency; SO, sphincter of Oddi; TTX, tetrodotoxin.
} 
define the manometric pressure characteristics of the opossum SO; (b) correlate SO electrical activity with intraluminal pressure phenomena; $(c)$ determine the relationship between SO electrical-mechanical activity and flow through the sphincter segment; and $(d)$ analyze the relationship between SO anatomy and its motor function.

\section{METHODS}

We studied 120 opossums weighing between 2.5 and $4.5 \mathrm{~kg}$. The animals were anesthetized with pentobarbital (100 mg i.p.) and maintained with intravenous pulse doses of 5-10 $\mathrm{mg}$ as needed. After anesthesia the animals were tied supine on a board. When warranted, a pressure ventilator maintained respiration. For drug administration we cannulated a femoral vein and infused $0.9 \%$ saline. A catheter in a femoral artery permitted continuous monitoring of blood pressure.

At laparotomy the extrahepatic bile duct system was readily visualized. The pancreatic duct (PD) joined the common bile duct (CBD) $\sim 3-4 \mathrm{~cm}$ above the duodenum. Beginning at the junction of the PD and CBD the duct showed overt thickening and exhibited spontaneous rhythmic contractions that propagated toward the duodenum. This segment of thickened duct in the opossum has been classified as the sphincter of Oddi (4). Entry of the SO into the proximal duodenum was marked by a thin slit rather than a papilla. The CBD and PD were thin walled and did not show evidence of contractile activity. After ligation of the cystic duct, two small polyvinyl (PV) catheters were tied into the right and left hepatic duct, respectively. These catheters were inserted into the proximal duodenum and served to bypass bile around the CBD and SO segments.

SO manometry was done using different catheters. For single-lumen PV catheters, a side-hole orifice was made 3 $\mathrm{mm}$ from the catheter tip. We used three different PV catheters of 1.6-, 1.0-, and $0.6-\mathrm{mm}$ o.d. The corresponding i.d. were $0.8,0.5$, and $0.31 \mathrm{~mm}$. Additionally, we used a multilumen PV catheter of $1.0-\mathrm{mm}$ o.d. that was extruded as a single catheter (Dural Plastics and Engineering, Ltd., Dural, New South Wales, Australia) with three lumens, each 0.32$\mathrm{mm}$ i.d. Three side-hole recording orifices were made at 5$\mathrm{mm}$ intervals beginning $3-\mathrm{mm}$ proximal to the catheter tip. For subsequent localization, we marked the distal $4 \mathrm{~cm}$ of each catheter with rings of black ink spaced at 2-mm intervals. To avoid undue resistance to fluid flow, each catheter was flared to a larger diameter beginning $8 \mathrm{~cm}$ from its tip. During manometry, each catheter lumen was infused with water at $0.1 \mathrm{ml} / \mathrm{min}$ using a minimally compliant hydraulic infusion system (12). Abrupt occlusion of the catheter recording orifices gave a pressure rise of $>300 \mathrm{~mm} \mathrm{Hg}$ in 1 s. During infusion the base-line resistance for different catheters ranged from 1 to $15 \mathrm{~mm} \mathrm{Hg}$ and was set to zero. Recordings were obtained using an 8-channel Beckman polygraph recorder (Beckman Instruments, Inc., Fullerton, CA).

For SO pull-throughs the recording catheter was inserted via the $C B D$ in 30 animals and via the $P D$ in 10 animals. Using single-lumen catheters of different outer diameters, pull-throughs across the SO were done by passing the catheter orifice into the duodenum and withdrawing it across the SO in $1-\mathrm{mm}$ increments, pausing $5 \mathrm{~min}$ at each station. In 27 animals, recording with the triple-lumen catheter was done with the distal recording orifice positioned $1 \mathrm{~cm}$ above the SO-duodenal junction. In 15 animals autonomic neural blockade was achieved by tetrodotoxin (TTX) (80-100 $\mu \mathrm{g}$ i.v.) during recording with the triple-lumen catheter $(8,13)$.

In seven animals a large endhole catheter of $2-\mathrm{mm}$ o.d. and $1.2 \mathrm{~mm}$ i.d. was advanced into the SO until resistance was encountered in the distal few millimeters of the SO segment. The catheter was then withdrawn $2 \mathrm{~mm}$ and secured in place by a tie $2-3 \mathrm{~mm}$ above the catheter tip (14). Continuous recordings were obtained while the catheter was infused at $0.1 \mathrm{ml} / \mathrm{min}$. We then administered amyl nitrite by inhalation and bethanechol $30 \mu \mathrm{g} / \mathrm{kg}$ i.v. to determine the effects of these agents on resistance to flow across the terminal portion of the SO segment.

Electrom yographic recordings were obtained from 35 animals. Monopolar electrodes were made of either silver wire insulated with teflon or nichrome wire insulated with trimel. The terminal $1 \mathrm{~cm}$ of each electrode was scraped free of insulation. Subsequently, the bared area was sutured through the SO muscularis. In each animal three to five electrodes were positioned at $5-\mathrm{mm}$ intervals along the SO segment. An electrode was also positioned on the duodenum, opposite the SO-duodenal junction. In five animals an electrode was positioned on the CBD. We inserted an indifferent electrode into the thigh musculature. To search for slow waves we used an $0.16 \mathrm{~Hz}$ low-frequency cut-off filter and a $30 \mathrm{~Hz}$ high-frequency cut-off filter. A low frequency cut-off of 5.3 $\mathrm{Hz}$ was used to record spike bursts. In five animals concurrent recordings of SO pressure and electrical activity were obtained by positioning a triple-lumen manometric catheter within the SO and suturing an electrode at the same level as each recording orifice.

During SO electromyography we determined the effect of fluid flow on SO spike burst frequency (SBF). We altered CBD inflow by adjusting the height of a saline reservoir and monitored flow by a drop counter $(60 \mathrm{gtt} / \mathrm{ml})$. We also altered SO spike burst frequency with drugs and evaluated the effect on the drop rate. The frequency of SO spike bursts was decreased by amyl nitrite inhalation or histamine 20 $\mu \mathrm{g} / \mathrm{kg}$ i.v. In five animals, a small balloon catheter $(2 \mathrm{~F})$ was passed into the SO and its balloon distended to a diameter of $4 \mathrm{~mm}$.

Cineradiographic recordings of SO motor activity were obtained at a frame rate of $15 / \mathrm{s}$ in six animals. Each animal had three to five monopolar electrodes spaced at 5-mm intervals along the SO and one in the duodenum. Water soluble contrast medium (Renografin 30\%) was dripped into the CBD from a reservoir while drop rate was monitored with a drop counter. During each study we used several different reservoir heights and flow rates. About 10-12 cineradiography sequences, lasting 30-90 s were obtained in each animal while simultaneous recordings were made of SO electrical activity and CBD inflow of contrast medium. During some sequences a single-lumen catheter of $0.6-\mathrm{mm}$ o.d. was inserted into the SO to record pressure. The cineradiography and polygraph recordings were synchronized using a dual event marker (15). Pharmacologic agents were given during some of the cineradiographic studies.

Eight SO specimens were examined histologically. Each specimen was fixed in formalin at its in vivo length. Cross sections, cut at 2-mm intervals, were stained with hematoxylin and eosin or Movat's stain.

For scoring manometric tracings, pressures obtained on SO pull-throughs were referenced to atmospheric pressure as zero. SBF of the SO electrical recordings was measured in number of spike bursts per minute. For sequences evaluating drug effects, SBF was scored in 1-min intervals for $3 \mathrm{~min}$ before and $5 \mathrm{~min}$ after drug administration. When evaluating the effect of flow rate on the frequency of SO 
spike bursts, SBF was scored for a 5-min interval beginning 3 min after a change in flow rate. From the cineradiographic recordings, the time and appearance of SO peristaltic stripping waves was correlated with the electromyographic and manometric tracings. For 35 individual SO peristaltic sequences, passage of the inverted- $\mathrm{V}$ tail of the contrast bolus by respective electrode recording sites was correlated with the timing of spike burst activity at the recording sites. We also evaluated the relationship of SO pressure phenomena and passage of the contrast bolus. The volume of contrast medium swept from the SO segment during a SO peristaltic sequence was determined using a planimetric method (16). Tests for statistical significance were done using the unpaired and paired Student's $t$ test. Averaged values in the text are given as mean $\pm 1 \mathrm{SE}$ unless otherwise indicated.

\section{RESULTS}

\section{Manometric findings}

Base-line pressure. We analyzed SO pull-throughs done with an $0.6-\mathrm{mm}$ o.d. catheter in $1-\mathrm{mm}$ increments from the duodenum to CBD for 30 animals and from the duodenum to PD for 10 animals. For all 40 animals the SO segment measured $3.5 \pm 0.6 \mathrm{SD} \mathrm{cm}$ in length. Basal pressure in the SO segment proximal to the SOduodenal high pressure zone (HPZ) measured $4 \pm 1 \mathrm{~mm}$ $\mathrm{Hg}$ above base-line duodenal pressure. CBD and PD pressure, respectively, measured $4 \pm 1 \mathrm{~mm} \mathrm{Hg}$ and $6 \pm 2$ $\mathrm{mm} \mathrm{Hg}$ above duodenal pressure. With the abdomen open, intraduodenal pressure equalled atmospheric pressure.

In all 40 animals a distinct $\mathrm{HPZ}, 5 \pm 0.4 \mathrm{SD} \mathrm{mm}$ in length, was recorded at the caudad end of the SO segment (Fig. 1). When withdrawing the catheter into the $\mathrm{CBD}$, an inconstant $\mathrm{HPZ}$ measuring $5 \pm 0.6 \mathrm{~mm}$ in length was recorded at the proximal margin of the SO in 16 of 30 animals. The proximal margin of the SOCBD HPZ was a few millimeters above the observed junction of the CBD and PD. When the catheter was positioned in the $\mathrm{PD}$ and withdrawn through the SO segment, a short HPZ measuring $2 \pm 0.4 \mathrm{SD} \mathrm{mm}$ was present at the proximal SO margin in 5 of 10 animals. No transient relaxations of basal pressure were observed at any of the three HPZ described. Basal pressure in the reproducible HPZ at the SO-duodenal junction depended on manometric assembly diameter (Table I). For the 0.6-mm o.d. catheter, the SO-duodenal HPZ averaged $18 \pm 3 \mathrm{~mm} \mathrm{Hg}$ above duodenal pressure and $14 \pm 3 \mathrm{~mm} \mathrm{Hg}$ above basal SO pressure (Fig. 2). Increases in the catheter's outer diameter above $0.6 \mathrm{~mm}$ caused significant increases in basal pressure of the SO-duodenal HPZ. For 16 animals with a detectable HPZ at the SO-CBD junction, the HPZ pressures averaged $2 \pm 2 \mathrm{~mm} \mathrm{Hg}$ above CBD pressure and showed no correlation with the catheters' outer diameter. Pressure at the SO-PD HPZ recorded with the $0.6-\mathrm{mm}$ o.d. catheter in 5 of 10 animals averaged $10 \pm 7 \mathrm{~mm} \mathrm{Hg}$ above PD pressure.

When resistance to flow across the SO-duodenal HPZ was recorded in seven animals by an infused end-hole catheter located just proximal to the HPZ, we obtained a pressure of $6 \pm 1 \mathrm{~mm} \mathrm{Hg}$ above duodenal pressure. This resistance to flow across the terminal SO HPZ showed no evidence of intermittent spontaneous relaxations. Amyl nitrite inhalation reduced the control pressure to $3 \pm 1 \mathrm{~mm} \mathrm{Hg}(P<0.05)$. In contrast, bethanechol increased terminal SO resistance to $25 \pm 7$ $\mathrm{mm} \mathrm{Hg}(P<0.05)$. Pressure resistance of the SO-duodenal HPZ was not altered by TTX.

Phasic contractions. The dominant feature of the

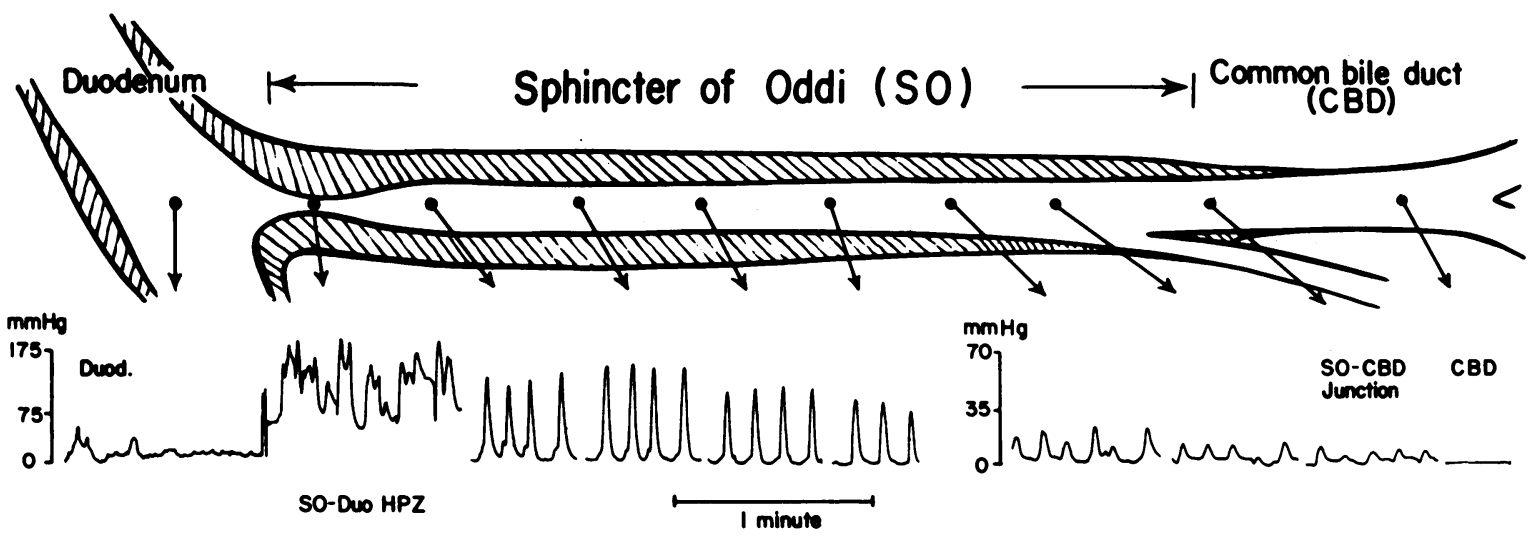

Figure 1 Station pull-through across the SO segment using a 0.6- $\mathrm{mm}$ diam catheter. Sample segments of tracing are shown for the sites indicated by dots. A HPZ is seen only in the distal SO segment. This HPZ did not exhibit transient relaxations to duodenal pressure. Phasic pressure waves occurred along the entire SO segment, but not in CBD. The phasic SO pressure waves are of maximal amplitude in the distal half of the SO and the amplitude decreases progressively toward the proximal SO. 
TABLE I

Effect of Catheter Diameter on SO HPZ

\begin{tabular}{cccc}
\hline & \multicolumn{3}{c}{ SO HPZ } \\
\cline { 2 - 4 } Catheter o.d. & SO-Duodenum & SO-CBD & SO-PD \\
\hline$m m$ & & & \\
1.6 & $(6) 75 \pm 10$ & (6) $6 \pm 4$ & - \\
1.0 & $(33) 40 \pm 5$ & (10) $4 \pm 3$ & - \\
0.6 & $(40) 18 \pm 3$ & (30) $2 \pm 2$ & (10) $10 \pm 7$ \\
\hline
\end{tabular}

Values represent mean $\pm 1 \mathrm{SE} \mathrm{mm} \mathrm{Hg}$. The numbers in parentheses denote the number of animals studied.

SO manometric tracings was phasic SO contractions (Fig. 1). These SO contractions were independent of duodenal motor activity. The SO contractions occurred spontaneously at a frequency of $4 \pm 1 / \mathrm{min}$ and were recorded along the entire SO segment. Amplitude of the phasic SO contractions was maximal in the distal half of the SO, ranging from 100 to $200 \mathrm{~mm} \mathrm{Hg}$, and progressively decreased to low values toward the proximal SO margin (Fig. 2). No phasic contraction waves were recorded in either the CBD or PD. The duration of the phasic pressure waves was $5 \pm 1 \mathrm{~s}$. Recordings from the triple-lumen catheter showed that the phasic contraction waves always originated near the proximal SO margin. About $80 \%$ of the contraction waves propagated the entire length of the SO segment to the duodenum (Fig. 3). The remaining $20 \%$ either did not propagate or propagated incompletely. By visual inspection, SO waves that propagated the full length of the SO segment were of greater amplitude than those waves that showed incomplete propagation. Propagation velocity of the antegrade peristaltic pressure waves ranged from 0.5 to $2.0 \mathrm{~cm} / \mathrm{s}$, with a mean of $1.1 \pm 0.8 \mathrm{SD} \mathrm{cm} / \mathrm{s}$. Retrograde SO contractions or isolated contractions in the middle or distal SO were not observed. In 15 animals given TTX, the nerve toxin failed to abolish the spontaneous SO contractions. After TTX, however, SO contraction frequency de-

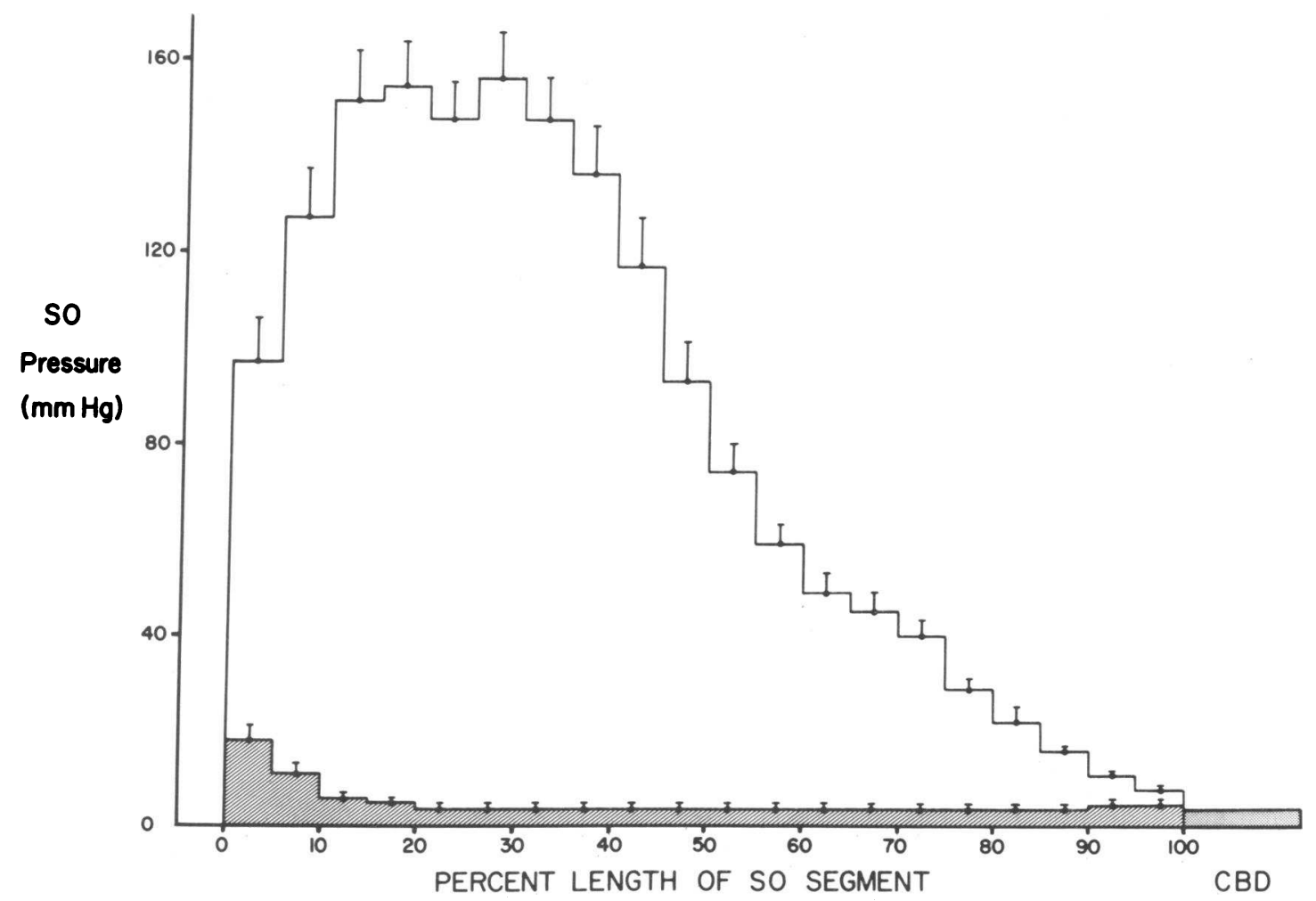

Figure 2 Composite of SO pressure values from 30 animals. SO pull-throughs were done in $1-\mathrm{mm}$ increments using an infused catheter of $0.6-\mathrm{mm}$ o.d. Data are normalized by plotting SO length in percent. The shaded area indicates base-line pressure across the SO segment and in CBD. A discreet HPZ is seen in the distal portion of the SO segment. A feeble HPZ is observed at the proximal SO margin. The open area of the histogram indicates the amplitude of phasic SO contractions. The phasic SO pressure waves were of maximal amplitude in the distal half of the SO and decreased progressively toward the proximal SO. 


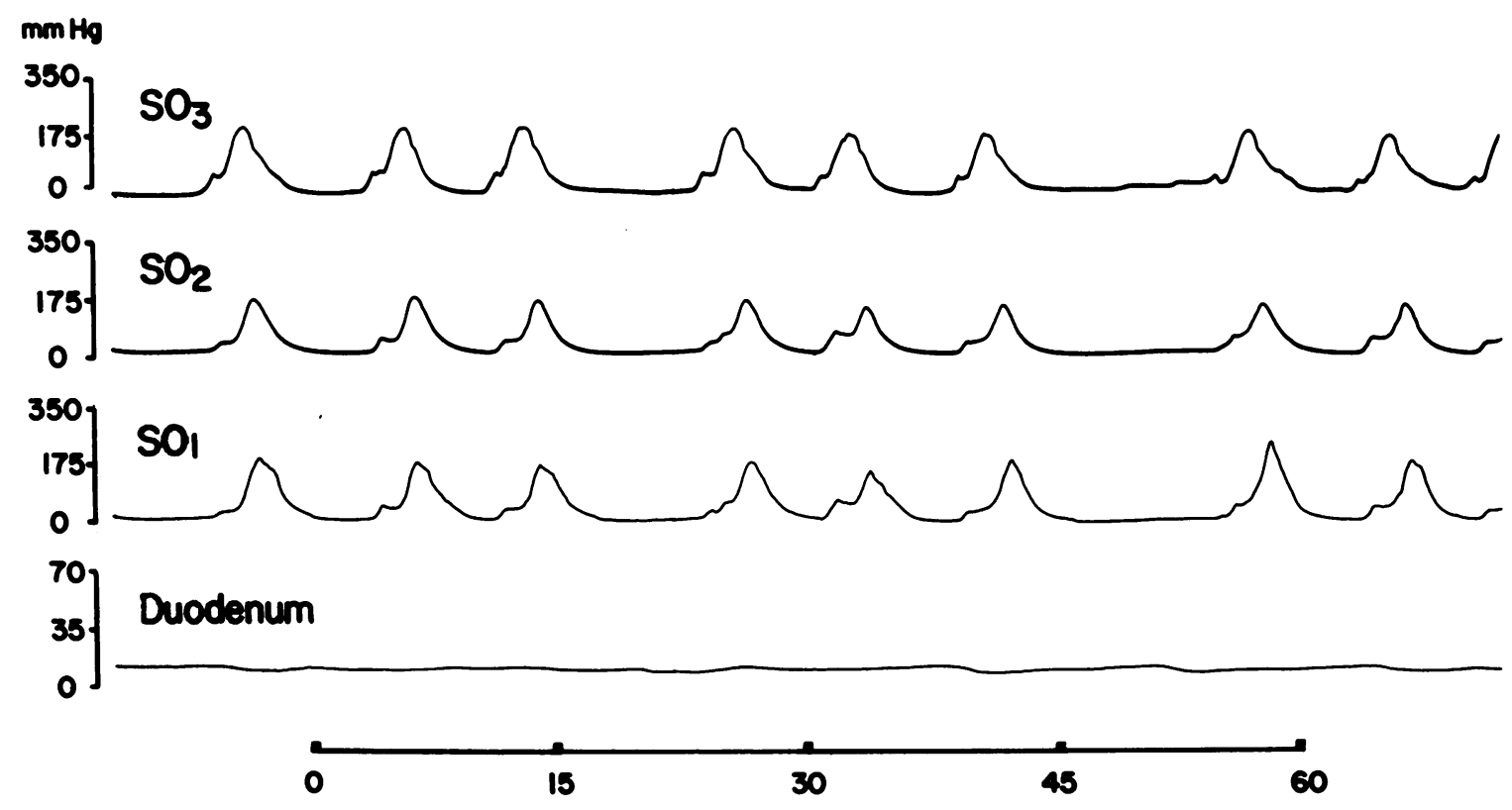

TIME IN SECONDS

FIGURE 3 Manomeric recording of phasic SO contractions. Each lumen of a triple-lumen catheter of $1.0-\mathrm{mm}$ o.d. was infused with water at $0.1 \mathrm{ml} / \mathrm{min}$ and the three recording sites were spaced at $5-\mathrm{mm}$ intervals. Each phasic contraction begins proximally and propagates as a peristaltic contraction toward the duodenum. Each pressure complex is characterized by a small initial hump followed immediately by a major pressure wave.

creased from $6.0 \pm 0.3$ to $2.0 \pm 0.2 / \mathrm{min}$ and contraction amplitude diminished from $52 \pm 8$ to $31 \pm 5 \mathrm{~mm} \mathrm{Hg}(P$ $<0.005)$.

\section{Myoelectric activity}

Myoelectric activity was recorded along the entire SO segment whereas the CBD showed no electrical activity. The SO myoelectrical recordings (Fig. 4) showed low amplitude undulations (slow waves) and rapid depolarizing oscillations (spike bursts). The low amplitude undulations varied in pattern among animals and in some instances from one interval to another in the same animal. During some intervals regular triphasic undulations were observed that appeared to be slow waves. During other recording intervals the myoelectric recording showed irregular low-amplitude oscillations that gave no recognizable pattern or the base line was flat. The duodenal recordings invariably showed regular rhythmic slow waves occurring at a frequency of $12-16 / \mathrm{min}$.

Spike bursts were observed on every SO myoelectric recording. In most cases spike bursts were clearly superimposed on slow waves. The spike bursts had a frequency of $8 \pm 2 \mathrm{SD} \mathrm{Hz}$ and lasted $1.0 \pm 0.4 \mathrm{SD}$ s. These spike bursts occurred spontaneously at a rate of 2-8/ min. Generally the interval between spike bursts was relatively uniform, but in some instances the spike bursts were organized into doublets or triplets. The SO spike bursts were not related to either duodenal slow waves or duodenal spike bursts. The SO spike bursts invariably originated in the proximal SO (Fig. 4). About $80 \%$ of the spike bursts propagated caudad in a peristaltic sequence over the entire length of the SO segment. The remaining $20 \%$ propagated only part of the way down the SO segment or did not propagate at all. Generally the spike bursts that did not propagate were of lower amplitude than those that propagated. The velocity of caudad propagation of the spike bursts was $1.0 \pm 0.3 \mathrm{SD} \mathrm{cm} / \mathrm{s}$. A few instances of cephalad propagation of the SO spike bursts were observed in a single animal.

\section{Correlation between myoelectric and manometric activity}

On inspection, every manometric contraction was associated with an underlying spike burst and each spike burst was accompanied by a pressure wave. The onset of the electrical spike burst preceded the onset of the corresponding manometric pressure wave by $1.0 \pm 0.2 \mathrm{SD}$ s. Visual observation suggested a rough 


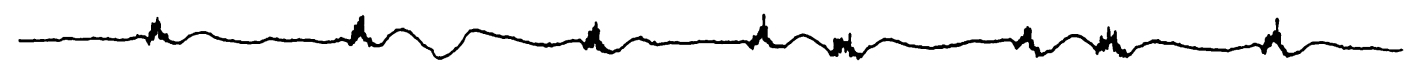

SO2

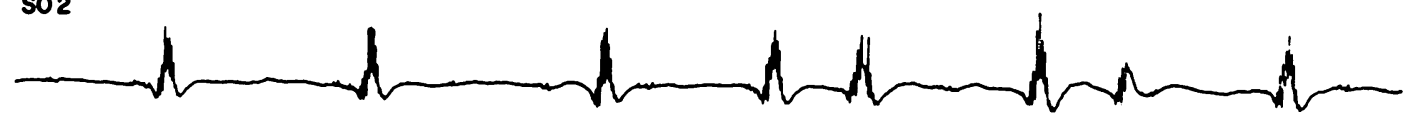

\section{sol}

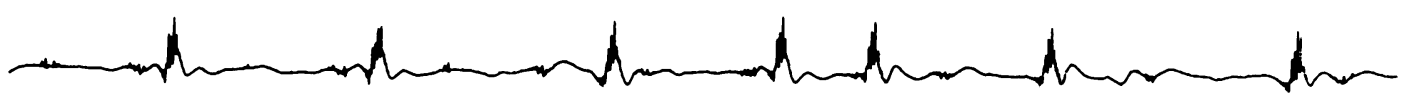

Duodenum

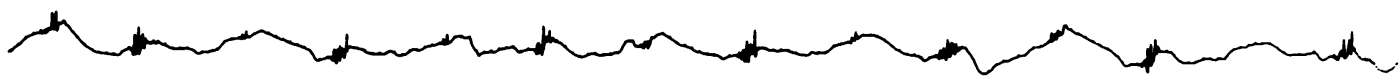

$\longmapsto 30$ ecconds

FIGURE 4 Myoelectric activity recorded from the SO and duodenum. Three electrodes are spaced along the SO at $5-\mathrm{mm}$ intervals. Regular slow waves occurring at a rate of $12 / \mathrm{min}$ and intermittently accompanied by spike bursts are observed in the duodenum. Slightly irregular triphasic SO slow waves are accompanied by spike bursts. The rhythmic SO spike bursts are observed at all three recording sites. The spike bursts originate in the proximal SO and propagate toward the duodenum. The SO spike bursts have no relationship to electrical activity of the duodenum.

\section{Drop}

counter

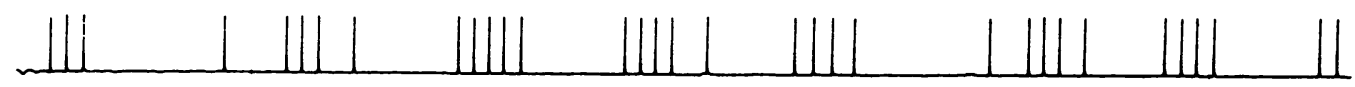

$\mathrm{SO}_{2}$

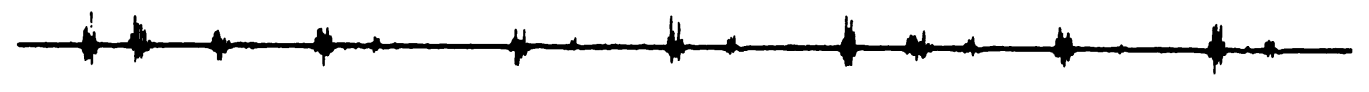

so,

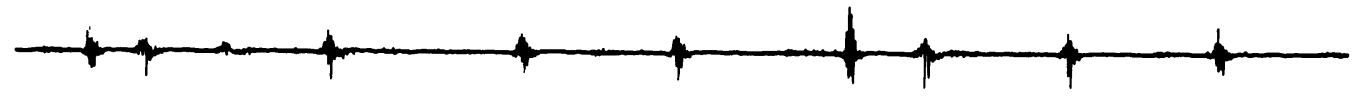

Duo
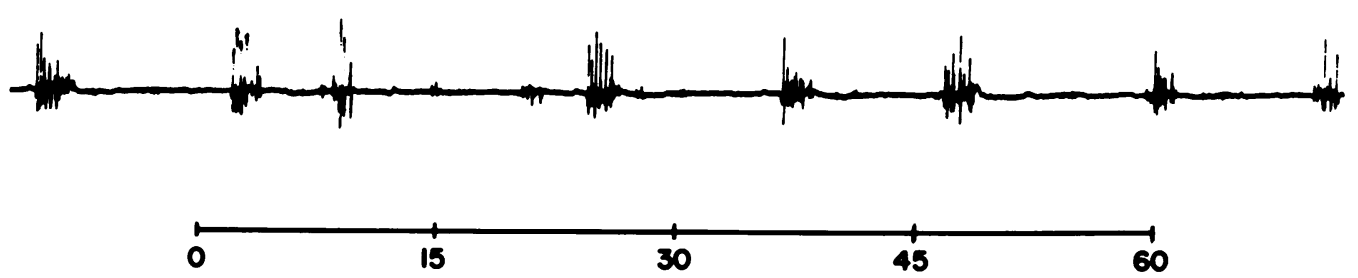

TIME IN SECONDS

Figure 5 Relationship of SO spike bursts to CBD flow. SO myoelectric activity was recorded by two electrodes spaced $1 \mathrm{~cm}$ apart while CBD inflow of saline was recorded by a drop counter $(60 \mathrm{gtt} / \mathrm{ml})$. Transient interruptions in CBD flow correspond to SO spike burst activity. The spike bursts immediately precede SO manometric pressure waves (data not shown) that are $\sim 5 \mathrm{~s}$ in duration. 
TABLE II

Effect of Drugs on SO SBF and Flow Rate

\begin{tabular}{|c|c|c|c|c|c|c|c|}
\hline \multirow[b]{2}{*}{ Drug } & \multirow{2}{*}{$\begin{array}{c}\begin{array}{c}\text { No. of } \\
\text { observations }\end{array}\end{array}$} & \multicolumn{2}{|c|}{ Control } & \multicolumn{2}{|c|}{ Post drug } & \multicolumn{2}{|c|}{ Calculated flow } \\
\hline & & Frequency & Drop rate & Frequency & Drop rate & Expected & Difference \\
\hline & & $S B / \min$ & $g t t / \min$ & $S B / \min$ & $g t t / \min$ & \multicolumn{2}{|c|}{$g t t / \min$} \\
\hline Histamine & 7 & $4.3 \pm 0.4$ & $12 \pm 4$ & $0 \pm 0^{\circ}$ & $25 \pm 5^{\circ}$ & $20 \pm 5$ & $5 \pm 07 \ddagger$ \\
\hline Amyl nitrite & 5 & $4.0 \pm 0.6$ & $10 \pm 3$ & $1 \pm 0.6^{\circ}$ & $28 \pm 5^{\circ}$ & $16 \pm 4$ & $12 \pm 3 \ddagger$ \\
\hline
\end{tabular}

- Differed from control value $(P<0.05)$.

† Significant difference of measured flow from estimated flow $(P<0.05)$.

SB, spike hurst.

correlation between the magnitude of an electrical spike burst and the amplitude of the associated manometric pressure wave.

Flow measurements. Frequency of the phasic SO contractions was influenced by SO distension. At a reservoir height of $15 \mathrm{~cm}$, the SO opening pressure was not exceeded. In the absence of flow the SO contraction frequency was $1.7 \pm 0.2 / \mathrm{min}$. A reservoir height of 20 $\mathrm{cm}$ increased CBD inflow to $0.3 \pm 0.1 \mathrm{ml} / \mathrm{min}$ ( $P$ $<0.05)$ and SO contraction frequency rose significantly to $3.2 \pm 0.3 / \mathrm{min}(P<0.001)$. SO distension by balloon inflation also increased the rate of SO contractions. Balloon inflation to $4 \mathrm{~mm}$ in the proximal SO increased SBF from $4 \pm 0.4$ to $16 \pm 1.5 / \mathrm{min}$ ( $P$ $<0.001$ ). Balloon inflation in the CBD caused no change in SO SBF. SO SBF was also affected by the presence of an indwelling luminal catheter. In 11 animals without an indwelling SO catheter and bile flow bypassed into the duodenum, SO SBF was $3 \pm 0.4 / \mathrm{min}$. By comparison, in 15 animals with a 1.0 -mm o.d. manometric catheter positioned in the SO, the SO SBF was $6 \pm 0.5 / \mathrm{min}(P<0.001)$.

With each SO contraction, CBD inflow stopped transiently for 4-6 s (Fig. 5). Abolition of the SO contractions with histamine or amyl nitrite caused a significant increase in the rate of CBD inflow (Table II). The increased rate of flow, however, could only be partially accounted for by the abolition of SO contractions. Calculated estimates of CBD flow that included the anticipated flow for the abolished intervals of SO contraction, were significantly less than the obtained values.

Cineradiographic findings. In each of six animals cineradiography demonstrated spontaneous SO peristaltic stripping waves. The waves started in the proximal SO and generally propagated the entire SO length, thereby emptying all contrast medium in the SO into the duodenum. During SO peristalsis and emptying, a narrowed segment, 4-5 $\mathrm{mm}$ in length and 0.5$0.7 \mathrm{~mm}$ in width, was observed in the distal portion of the SO segment. The transverse diameter of the distended SO segment above the terminal narrowing measured 1.6 $\pm 0.5 \mathrm{SD} \mathrm{mm}$. Calculation of SO stroke volume for individual complete SO peristaltic sequences gave values ranging from 0 to $0.05 \mathrm{ml}$. The stroke volume depended on the degree of SO filling at the onset of SO peristalsis. When the SO peristaltic waves propagated incompletely only a portion of the intraluminal SO contrast medium was emptied into the duodenum. Retrograde stripping waves were not observed.

A precise correlation existed between the SO peristaltic stripping waves observed on cineradiography,

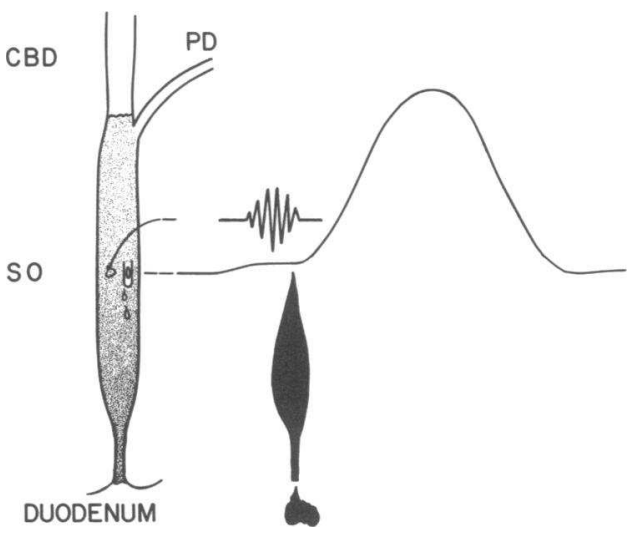

Figure 6 Schema showing the correlation between electromyographic, manometric, and cineradiographic recordings of a peristaltic contraction traversing the SO segment. The myoelectric and manometric tracings were obtained at the same SO level. During SO peristalsis the bolus tail passed the manometric recording site. A precise relationship was observed among the recordings of SO peristalsis obtained by the three recording methods. A myoelectric spike burst immediately precedes and slightly overlaps the onset of the major upstroke of the peristaltic pressure complex. The upstroke onset was essentially coincident with passage of the bolus tail. A small pressure hump occurs immediately before the major pressure wave. The cineradiographic studies demonstrated that this pressure hump was recorded from within the contrast bolus before the bolus tail reached the recording site. 
the SO spike bursts recorded by electromyography, and manometric pressure wave tracings from the SO lumen (Fig. 6). During SO peristalsis, passage of the inverted V-tip of the bolus tail by a given electrode occurred at $0.7 \pm 0.2 \mathrm{SD} s$ after the onset of the myoelectric spike burst occurred at the electrode. Passage of the bolus tail by the intraluminal SO manometric recording site was essentially coincident with the onset of the manometric pressure wave. Before passage of the major pressure complex, however, a modest pressure rise of 5-20 mm Hg occurred within the SO bolus as the peristaltic stripping wave stripped the SO segment (Fig. 3). Similar to the findings from the flow studies with saline, the flow of contrast medium into the CBD halted transiently in association with each SO peristaltic contraction sequence.

The SO dynamics for a CBD flow rate of $0.1 \mathrm{ml} /$ min provided by a reservoir height of $18 \mathrm{~cm}$ are shown in Fig. 7. In the example illustrated, SO SBF was 3/ min. During SO peristalsis, SO contents were actively emptied into the duodenum and passive CBD filling stopped briefly. After SO peristalsis was completed, passive CBD filling recommenced and the SO segment began to fill passively without emptying into the duodenum. The passive SO filling ended with the next SO peristaltic sequence and the cycle continued. At higher CBD flow rates the rate of passive SO filling increased and in some instances, eg., at a flow rate of $0.3 \mathrm{ml} /$ min, passive SO emptying was observed in addition to active SO emptying.

Cineradiographic studies were also obtained after giving drugs that altered the frequency of SO peristalsis. Histamine $(20 \mu \mathrm{g} / \mathrm{kg}$ i.v. $)$ and amyl nitrite inhalation abolished SO contractions and caused a substantial increase in CBD inflow. During this interval the entire SO segment including its narrowed terminal portion remained open while contrast medium flowed passively into the duodenum. Phenylephrine $(50 \mu \mathrm{g} /$ $\mathrm{kg}$ i.v. $)$ and bethanechol $(30 \mu \mathrm{g} / \mathrm{kg}$ i.v. $)$ increased the frequency of SO contractions and virtually abolished

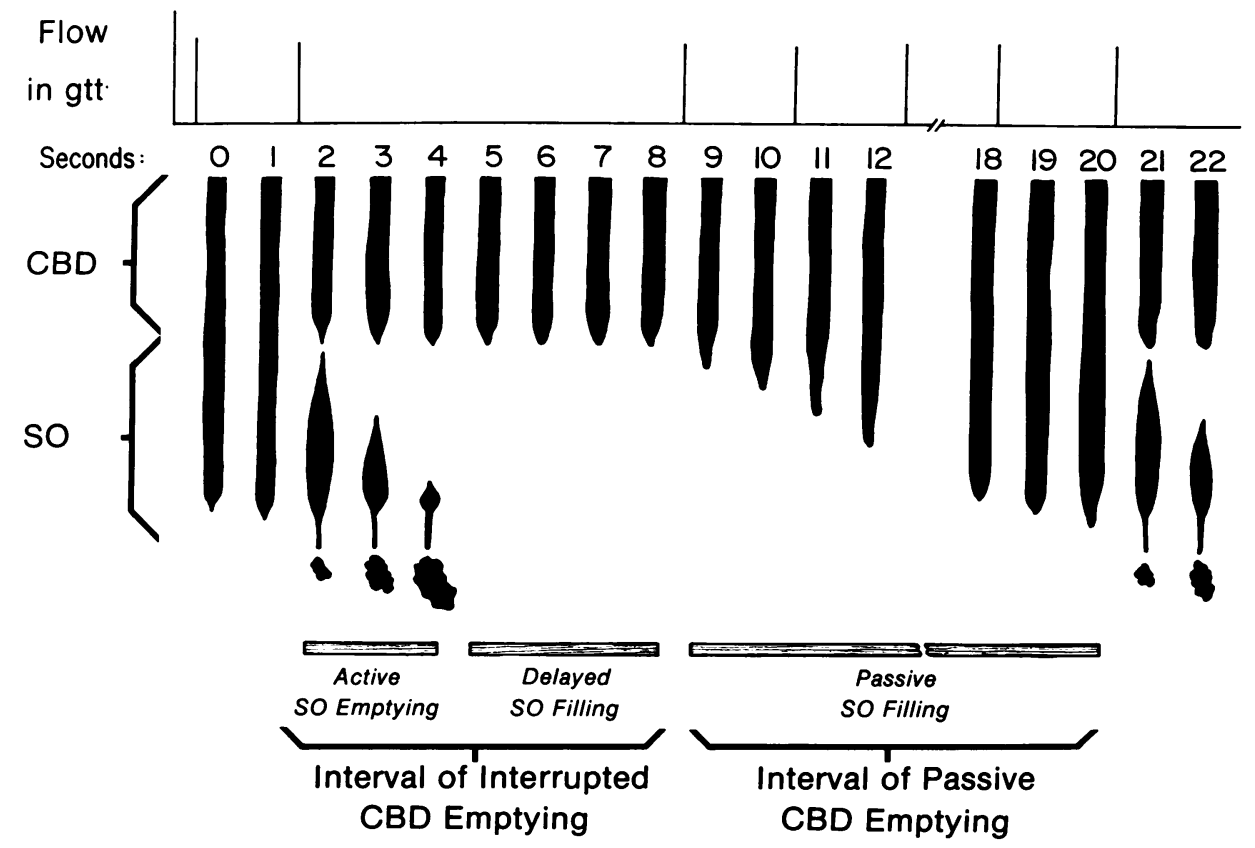

FiguRE 7 Diagramatic representation of bile duct emptying drawn from images of a cineradiographic recording. A catheter positioned in the proximal CBD for infusing contrast is not shown. Time in seconds is given above the images of contrast material. In this example, contrast medium flowed into the CBD at a rate of $\sim 6 \mathrm{gtt} / \mathrm{min}(0.1 \mathrm{ml} / \mathrm{min})$ from a reservoir. Before SO peristalsis, contrast flowed into the CBD, which then emptied passively into the SO segment. Thus, the drops indicate CBD outflow as well as CBD inflow. During a 2-s interval SO peristalsis actively expelled contrast from the SO segment into the duodenum. A zone of narrowing is observed in the distal SO segment. During passage of the SO contraction wave the SO segment was pinched off from the CBD, thereby interrupting passive CBD outflow into the SO segment. Following SO emptying, a short 4-s interval of delayed SO filling persisted until SO contraction was complete. When the pressure in the SO segment returned to its initial value after peristalsis, fluid flow from the CBD into the SO recommenced leading to passive SO filling during SO diastole. The cycle repeated itself with the onset of the next SO peristaltic contraction. 


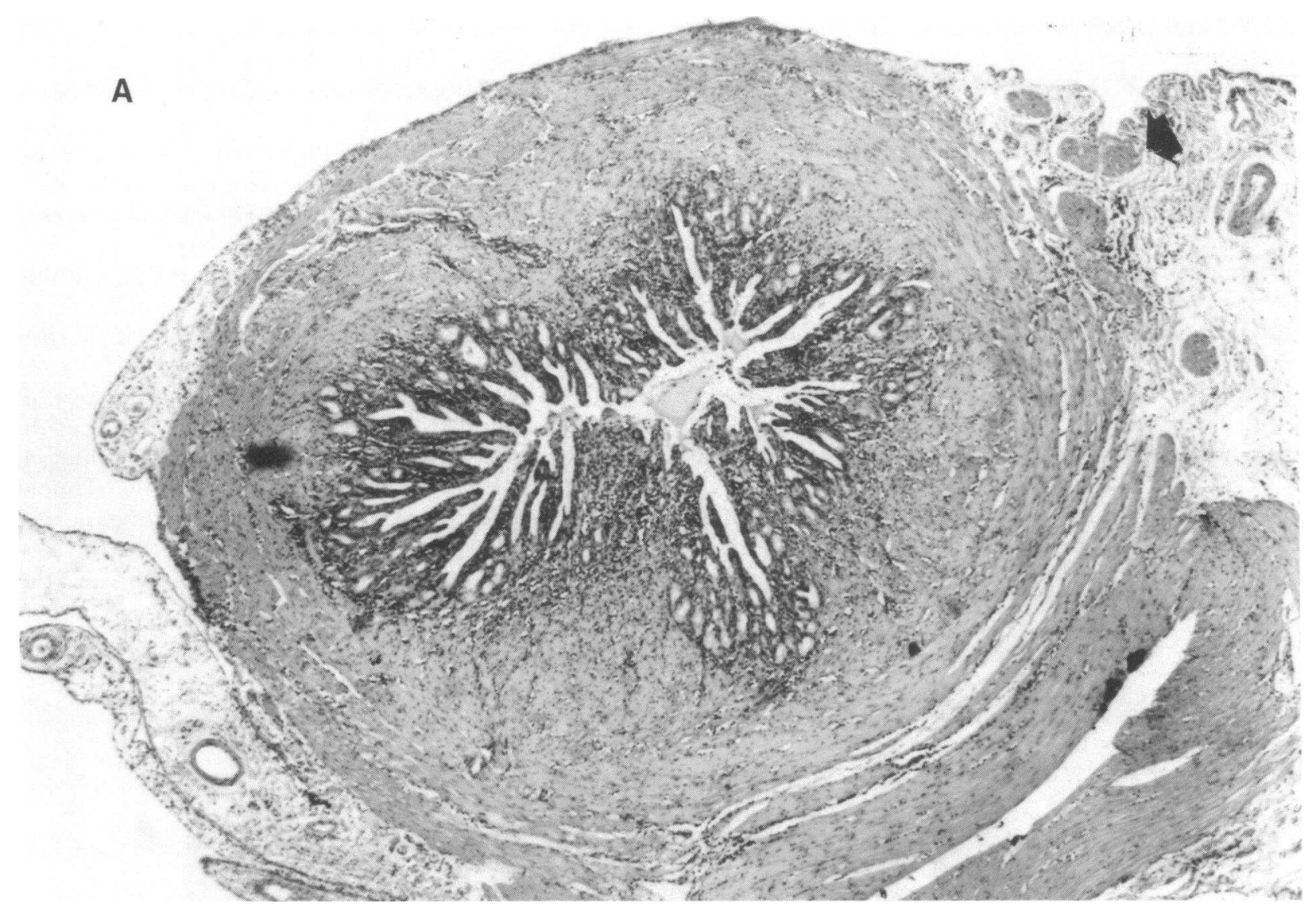

FIGURE 8 Histological sections through the opossum SO segment ( $\times 40$ magnification). (A) Section through terminal SO $2 \mathrm{~mm}$ above its entry into the duodenum. This level corresponds to the manometric HPZ in the distal SO. The mucosa is folded into frondlike projections. The musculature consists of an inner layer of longitudinal muscle surrounded by a prominent layer of circular muscle. A few scattered bundles of longitudinal muscle (arrow) are present external to the circular muscle. A large blood vessel is seen on the lower right. (B) Section through the proximal SO margin at the junction of the CBD and PD. Pancreas is seen on the lower right. Only a thin layer of circular muscle fibers surrounds the junction of the CBD (left) and PD (right). The thin septum between the CBD and PD is comprised of connective tissue stoma containing few muscle fibers.

SO filling and CBD inflow. During a 1-3-min interval after these latter agents the SO remained as a contracted empty segment.

Histological findings. The SO segment was characterized by three layers of smooth muscle comprised of a inner longitudinal layer, a middle circular layer, and an incomplete outer longitudinal layer. The thickest layer was the circular muscle layer. Thickness of circular muscle was maximal at the terminal $4-5 \mathrm{~mm}$ of the SO segment (Fig. 8A) and corresponded anatomically to the SO-duodenal HPZ recorded on manometry. The circular muscle layer gradually decreased in thickness towards the cephalad end of the SO segment. At the junction of the CBD with the PD, a thin layer of circular muscle enveloped both ducts (Fig. 8B) and extended a few millimeters along both ducts as a scanty layer of muscle. There was no evidence of thickened circular muscle to suggest a sphincter at the distal end of the CBD or PD. The inner longitudinal muscle layer, located immediately under the mucosa was interspersed amongst collagen fibers within the submucosa. The incomplete outer longitudinal muscle existed as scatterd bundles with no connection between the individual bands. Thickness of the longitudinal musculature diminished progressively from the caudad to cephalad end of the SO segment and was absent in both the CBD and PD. Smooth muscle of the SO was histologically separate from duodenal smooth muscle. The SO segment communicated with the duodenum through a small window in the duodenal muscle. Neural ganglia were present along the whole SO segment, but virtually absent in the CBD and PD. 


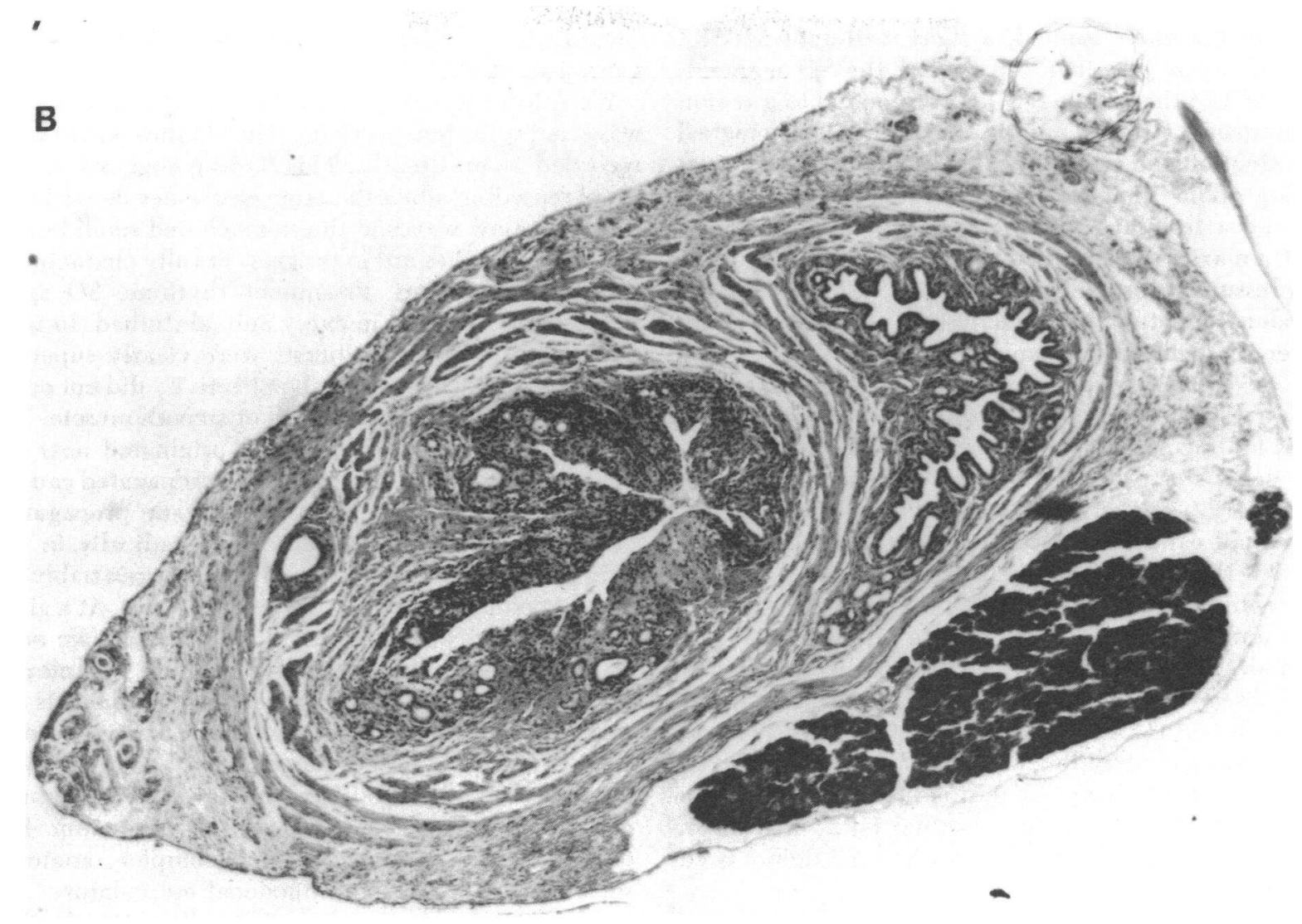

Figure 8 (Continued)

Ganglia cells were located mainly on the outer surface of the circular muscle layer and showed an increased density at the proximal SO margin. The SO segment was lined by columnar epithelium arranged in prominent fronds that projected into the lumen. The fronds were most prominent in the terminal narrow portion end of the SO segment and gradually decreased in size and number towards the cephalad end of the SO segment.

\section{DISCUSSION}

In this study we evaluated the morphology and motor function of the opossum SO. The major study findings were $(a)$ the opossum SO segment exhibits muscle thickening and spontaneous rhythmic contractions; $(b)$ a short HPZ, consisting of active and passive components, exists at the terminus of the SO segment and corresponds to a zone of anatomical narrowing; (c) manometric and myoelectric recordings show phasic contractile activity that originates near the cephalad SO margin and propagates caudad as a peristaltic sequence; $(d)$ SO distension increases the frequency of SO peristaltic contractions; and $(e)$ cineradiography reveals peristaltic SO stripping waves, corresponding to manometric pressure waves and myoelectric spike bursts, that actively empty the SO segment of contrast medium.

We have retained the term "sphincter of Oddi" used by Boyden (4) to designate the duct segment located between the CBD-PD junction and the duodenum in the opossum. This segment in the opossum is largely extraduodenal and measures $3-4 \mathrm{~cm}$ in length, compared with a $0.5-1.0 \mathrm{~cm}$ length in man (5). The SO segment in both species exhibits prominent phasic contractile activity. An alternative approach to the conventional terminology would be to call the SO segment a terminal muscular portion of the CBD.

Most manometric studies designed to evaluate the SO in experimental animals have yielded indirect recording of the pressure gradient across the SO segment or resistance to flow rather than direct pressure recordings (17-20). Direct intraluminal recording from the opossum SO demonstrated two major findings: (a) a short HPZ at the distal end of the SO segment, and (b) rhythmic phasic contraction waves along the entire SO segment. The SO segment, therefore, exhibits both tonic and phasic pressure phenomena. 
In all opossums studied, a short well-defined HPZ existed in the terminal 4-6 $\mathrm{mm}$ of the SO segment. The HPZ of the terminal SO corresponded to a region of increased circular muscle thickness on histological examination and a zone of luminal narrowing on cineradiographic images. The cineradiographic studies showed a terminal SO narrowing 4-5 $\mathrm{mm}$ in length with a maximal diameter of $\sim 0.7 \mathrm{~mm}$. The magnitude of pressure recorded in the terminal HPZ at the SOduodenal junction was directly related to the catheter outer diameter. For example, a catheter of $1.6-\mathrm{mm}$ o.d. gave pressure values $>50 \mathrm{~mm} \mathrm{Hg}$ whereas the 0.6$\mathrm{mm}$ o.d. catheter recorded values of $\sim 15-20 \mathrm{~mm} \mathrm{Hg}$. This finding suggests that a substantial component in the genesis of pressure recorded from within the terminal HPZ is passive resistance to stretch caused by the small caliber of the terminal SO. A second component that contributes to the terminal HPZ is active sphincter tone. This conclusion is based on the finding that amyl nitrite, a smooth muscle relaxant, produced a modest but significant relaxation. However, we did not observe any spontaneous transient relaxations of the terminal HPZ as suggested in a previous study (6). This unexpected finding may be explained by two possibilities: (a) our instrumentation failed to detect spontaneous relaxations of the terminal $\mathrm{HPZ}$, or (b) transient relaxations of the SO terminal $\mathrm{HPZ}$ do not occur spontaneously.

Although a well-defined terminal HPZ was present in the SO of all animals. HPZ at the proximal SO recorded via CBD and PD pull-throughs were detected in only half the animals and were of low amplitude when present. When recorded, these feeble HPZ did not relax either spontaneously or after amyl nitrite. The histological sections showed a paucity of circular smooth muscle in the terminal portions of the CBD and PD adjacent to their junction with the SO segment. Functioning minisphincters in these areas were not suggested by cineradiography. Thus, our study results do not provide convincing evidence to support the presence of physiological sphincters at the CBD-SO and PD-SO junctions in the opossum as suggested in an earlier study (6).

The dominant manometric feature of the opossum SO was spontaneous rhythmic pressure waves that originated in the proximal SO and generally propagated the entire SO length. Manometric recordings from the normal human SO show mostly antegrade peristaltic pressure waves, albeit $\sim 15 \%$ of the contraction sequences in the human SO are retrograde (21). The fact that TTX did not abolish the phasic contractions in the opossum SO indicates that the contractions were myogenic in origin. The phasic SO contractions always originated near the proximal SO margin, thereby implying a pacemaker in this region (22). A high density of ganglion cells in the proximal SO suggests the possibility of neural control over a myogenic pacesetter.

Electrical recordings from the SO showed both slow wave and spike burst activity. The SO slow waves were recorded inconsistently. This finding suggests suboptimal recording albeit the same electrodes showed omnipresent slow waves in the stomach and small bowel. Additional studies are in progress to fully characterize the SO slow waves. Prominent rhythmic SO spike bursts were recorded in every animal studied. In most instances the SO spike bursts were clearly superimposed on slow waves. Spike burst activity did not occur in the CBD, a structure devoid of smooth muscle. The SO spike bursts virtually always originated near the proximal SO margin and generally propagated caudad over the entire SO segment. Retrograde propagation of the SO spike bursts occurred sporadically in one animal. A well-defined spike burst was invariably accompanied by a manometric pressure wave. At a given SO level, the spike burst signaled the upstroke onset of the underlying manometric pressure complex. A similar temporal relationship between spike bursts and pressure waves has been shown in the opossum esophagus (23). The SO spike bursts and manometric pressure waves had no relationship to electrical or mechanical activity recorded from the duodenum. Histological examination confirmed complete anatomic separation of the SO and duodenal musculature.

SO distension by fluid, an indwelling intraluminal catheter, or balloon distension all caused a significant increase in SBF. The physiological role of distension in regulating SO SBF remains to be determined. In a chronic preparation, the frequency of SO spike bursts ranges from $\sim 1$ to 8 in conscious opossums (24), sim: ilar to the range of values obtained here from anesthetized animals.

Studies measuring CBD flow of saline and subsequently contrast medium showed interruption of flow during SO peristaltic contractions. The periodic interruptions of flow lasted $\sim 4-6 \mathrm{~s}$, approximately the duration of the SO pressure waves. This seemingly paradoxical finding, observed also in other species (18, $25)$, has led some workers to conclude that the function of phasic SO contractions is to retard CBD outflow (25-27). When considered further, however, emptying of fluid from the CBD into the SO segment would not be expected during forceful SO contractions, even though the SO contractions propagate toward the duodenum. Namely, fluid cannot flow from a low pressure CBD into a high pressure SO segment undergoing contraction. Although the peristaltic SO contractions empty the SO segment, they cause an obligatory transient arrest of CBD outflow. This phenomenon is analogous to cardiac ventricular contractions that transiently interrupt atrial emptying. Because the peristaltic SO contractions evacuate the SO segment, they 
may in fact promote CBD outflow by rendering the SO segment empty for filling from the CBD between SO contractions. These conclusions are supported by the cineradiographic studies. Four major findings were evident from viewing the cineradiographic images. First, the CBD appeared as a passive tube without contractile activity. Secondly, the SO segment exhibited rhythmic systolic emptying and diastolic filling. During SO systole a peristaltic stripping wave emptied SO contents into the duodenum. The stroke volume of SO peristalsis ranged from 0 to $0.05 \mathrm{ml}$ of contrast medium, depending on the degree of SO filling during diastole. Thirdly, the distal SO funneled into a narrow zone $\sim 5 \mathrm{~mm}$ in length that appeared to act as a resistor to SO outflow. Lastly, no constriction was observed at the CBD-SO junction to suggest a sphincteric structure at this location.

The cineradiographic observations correlated with the findings from histological examination, electromyography, and intraluminal manometry. The passive CBD had negligible muscle and did not show phasic contractile activity. In contrast, the entire SO segment had a prominent circular muscle layer capable of generating peristaltic contractions. A precise relationship existed between the expression of SO peristaltic activity on the cineradiographic, myoelectric, and manometric recordings. Passage of the tail of intraluminal SO contrast medium, configured by the SO contraction, corresponded to upstroke onset of the peristaltic pressure complex. A similar relationship has been shown previously in the esophagus (15). In the SO, electrical spike bursts at a given level immediately preceded the onset of the manometric pressure complex and concurrent passage of the bolus tail. Thus, passage of a bolus tail through the SO on cineradiography is the equivalent of the myoelectric spike burst and onset of the manometric peristaltic pressure wave. Establishment of these interrelationships allows the investigator the option to monitor SO peristalsis by any one of the three recording methods. The terminal zone of SO narrowing observed on cineradiography corresponded to the short $\mathrm{HPZ}$ recorded on manometry. The muscularis of the terminal zone was thicker than elsewhere in the SO segment.

Some interpretation of the study findings is merited. The opossum SO segment appears to function as a smooth muscle pump linked in series with the passive CBD and PD conduits. Exclusive of gallbladder contractions, the only pressure head governing CBD outflow is the modest secretory pressure generated by the liver (28). Similarly, the force responsible for PD flow is pancreatic secretory pressure. Under basal conditions the normal CBD and PD to duodenal pressure gradients across the $\mathrm{SO}$ are $\sim 5-10 \mathrm{~mm} \mathrm{Hg}$ in a variety of animal species $(10,17,19)$. Even with CBD obstruction, maximal intraductal pressure does not rise much above $30 \mathrm{~mm} \mathrm{Hg}$ because net hepatic output of bile fails at $\sim 30 \mathrm{~mm} \mathrm{Hg}$ of pressure $(29,30)$. The dominant feature of the opossum SO is rhythmic peristaltic contractions that actively empty the SO segment. After the systolic contraction, the SO segment refills passively during its diastole by passive inflow from the CBD. The degree of diastolic SO filling depends on the magnitude of CBD pressure, the rate of CBD flow and the interval of SO diastole. SO filling may range from zero during rapid SO contractions of 10-12 min induced by excitatory drugs to $\sim 0.05 \mathrm{ml}$, which is the maximal intraluminal volume of the $S O$ segment when filled completely. During SO systole the peristaltic stripping wave ejects $\mathrm{SO}$ contents across the terminal zone of SO narrowing into the duodenum. As the forceful peristaltic contraction propagates through the SO, pressure within the intraluminal bolus rises $5-20 \mathrm{~mm}$ $\mathrm{Hg}$ to overcome the resistance of the terminal SO narrowing. SO peristalsis is capable of forcing fluid through the distal SO narrowing regardless of whether or not the terminal HPZ relaxes. Recordings with a catheter in the zone of narrowing failed to demonstrate a transient $\mathrm{HPZ}$ relaxation in coordination with $\mathrm{SO}$ peristalsis.

On occasional cineradiographic sequences passive SO emptying was observed when the rate of CBD outflow and duration of $\mathrm{SO}$ diastole combined to achieve complete SO filling before the next SO peristaltic contraction. Passive emptying of the SO occurred dropby-drop and lasted a short interval before the next SO systole. In this circumstance, pressure within the common channel of CBD and SO was sufficient to overcome the resistance of the terminal SO. Whether any passive emptying of the opossum SO occurs physiologically was not determined in this study.

In addition to expelling bile from the SO segment, a second function of SO peristalsis is to keep the terminal zone of SO narrowing free of obstructing material. Due to the small diameter of the terminal SO zone, small food particles, mucous or sludge, could occlude the terminal SO narrowing so that the modest pressure gradient generated by biliary secretion could not clear the obstructing material. SO peristalsis, however, is capable of generating a substantial pressure head to eject particulate material from the narrowed segment. Indeed, any SO distension caused by distal SO obstruction would serve to increase the frequency of SO peristalsis as a mechanism to overcome the obstruction. Thus, SO peristalsis appears to have an important housekeeping function of maintaining the patency of the terminal SO narrowing that would always be at risk for repeated episodes of obstruction if its patency were dependent solely on the minimal passive pressure gradient between the CBD and duodenum. In the human SO peristalsis might expel small calculi from the distal CBD into the duodenum.

$$
\text { Opossum Sphincter of Oddi }
$$


In this study, we endeavored to characterize and analyze the dynamics of SO motor function in the opossum. This model has two principle virtues in that the opossum SO is accessible for study and exhibits peristaltic contractions similar to those recorded in humans. Although findings from the opossum should not be extrapolated directly to the human SO, we anticipate that thorough investigation of the opossum model will give useful information for designing and analyzing investigative studies of the human SO.

\section{ACKNOWLEDGMENT}

This work was supported, in part, by National Institutes of Health grants AM 15540 and AM 27531.

\section{REFERENCES}

1. Lin, T. M. 1975. Actions of gastrointestinal hormones and related peptides on the motor function of the biliary tract. Gastroenterology. 69: 1006-1022.

2. LaMorte, W. W., D. J. Schoetz, Jr., D. H. Birkett, and L. F. Williams, Jr. 1979. The role of the gallbladder in the pathogenesis of cholesterol gallstones. Gastroenterology. 77: 580-592.

3. Becker, J. M., W. M. Duff, and F. G. Moody. 1981 Myoelectric control of gastrointestinal and biliary motility: a review. Surgery (St. Louis). 89: 466-477.

4. Boyden, E. A. 1937. The sphincter of Oddi in man and certain representative mammals. Surgery (St. Louis). 1: 25-37.

5. Boyden, E. A. 1957. The anatomy of the choledochoduodenal junction in man. Surg. Gynecol. Obstet. 104: 641652

6. Shelhamer, J. 1973. Physiology of the bile transport: manometric studies of common bile duct and sphincter of Oddi. Gastroenterology 64: 686a. (Abstr.).

7. Shaw, B. M., J. M. Becker, and F. G. Moody. 1979. Histaminergic responses of biliary sphincter in opossum. Surg. Forum. 30: 400-402.

8. Toouli, J., W. J. Dodds, R. Honda, and W. J. Hogan. 1981. Effect of histamine on motor function of opossum sphincter of Oddi. Am. J. Physiol. 4: G122-G128.

9. Csendes, A., A. Kruse, P. Funch-Jensen, M. J. Oster, J. Ornsholt, and E. Amdrup. 1979. Pressure measurements in the biliary and pancreatic duct systems in controls and in patients with gallstones, previous cholecystectomy, or common bile duct stones. Gastroenterology. 77: 1203-1210.

10. Geenen, J. E., W. J. Hogan, W. J. Dodds, E. T. Stewart, and R. C. Arndorfer. 1980. Intraluminal pressure recording from the human sphincter of Oddi. Gastroenterology. 78: 317-324.

11. Carr-Locke, D. L., and J. A. Gregg. 1981. Endoscopic manometry of pancreatic and biliary sphincter zones in man. Basal results in healthy volunteers. Dig. Dis. Sci. 26: 7-15.

12. Arndorfer, R. C., J. J. Stef, W. J. Dodds, J. H. Linehan, and W. J. Hogan. 1977. Improved infusion system for intraluminal esophageal manometry. Gastroenterology. 73: $23-27$.
13. Dent, J., W. J. Dodds, W. J. Hogan, R. C. Arndorfer, and B. C. Teeter. 1980. Effect of cholecystokinin-octapeptide on opossum lower esophageal sphincter. Am. J. Physiol. 239: G230-G235.

14. Behar, J., and P. Biancani. 1980. Effect of cholecystokinin and the octapeptide of cholecystokinin on the feline sphincter of Oddi and gallbladder: mechanisms of action. J. Clin. Invest. 66: 1231-1239.

15. Dodds, W. J., E. T. Stewart, D. Hodges, and F. F. Zboralske. 1973. Movement of the feline esophagus. associated with respiration and peristalsis. An evaluation using tantalum markers. J. Clin. Invest. 52: 1-13.

16. Silva, G. S. 1949. A simple method for computing the volume of the human gallbladder. Radiology. 52: 94102.

17. Watts, J. M., and J. E. Dunphy. 1966. The role of the common bile duct in biliary dynamics. Surg. Gynecol. Obstet. 122: 1207-1218.

18. White, T. T., H. Waisman, D. Hopton, and H. Kavlie. 1972. Radiomanometry, flow rates, and cholangiography in the evaluation of common bile duct disease. Am. J. Surg. 123: 73-79.

19. Persson, C. G. A. 1973. Dual effects on the sphincter of Oddi and gallbladder induced by stimulation of the right great splanchnic nerve. Acta. Physiol. Scand. 87: 334343.

20. Scott, G. W., R. E. Smallwood, and S. Rowlands. 1975. Flow through the bile duct after cholecystectomy. Surg. Gynecol. Obstet. 140: 912-918.

21. Toouli, J., J. E. Geenen, W. J. Hogan, W. J. Dodds, and R. C. Arndorfer. 1982. Sphincter of Oddi motor activity: a comparison between patients with common bile duct stones and controls. Gastroenterology. 82: 111-117.

22. Helm, J. F., J. Christensen, W. J. Dodds, and S. Sarna. 1981. In vitro contractile activity of opossum sphincter of Oddi (SO) muscle rings. Gastroenterology. 80: $1171 a$. (Abstr.).

23. Goyal, R. K., and J. S. Gidda. 1981. Relation between electrical and mechanical activity in esophageal smooth muscle. Am. J. Physiol. 3: G305-G311.

24. Honda, R., J. Toouli, W. J. Dodds, W. J. Hogan, S. Sarna and Z. Itoh. 1982. Relationship of sphincter of Oddi spike bursts to gastrointestinal myoelectric activity in conscious opossums. J. Clin. Invest. 69: 770-778.

25. Ishioka, T. 1959. Electromyographic study of the choledochoduodenal junction and duodenal wall muscle. Tohoku J. Exp. Med. 70: 73-84.

26. Ono, K., N. Watanabe, K. Suzuki, H. Tsuchida, Y. Sugiyama, and $M$. Abo. 1968. Bile flow mechanism in man. Arch. Surg. 96: 869-874.

27. Becker, J. M., F. G. Moody, and A. R. Zinsmeister. 1982. The effect of gastrointestinal hormones on the biliary sphincter of the opossum. Gastroenterology. 82: 13001307.

28. Hess, W. 1965. Surgery of the Biliary Passages and the Pancreas. D. Van Nostrand Co., Inc., Princeton, NJ.

29. Hallenbeck, G. A. 1967. Biliary and pancreatic intraductal pressures. Handb. Physiol. 2(Sect. 6, Alimentary Canal Secretion): 1007-1025.

30. Strasberg, S. M., B. C. Dorn, R. N. Redinger, D. M. Small, and R. H. Egdahl. 1971. Effects of alteration of biliary pressure on bile composition-a method for study: primate biliary physiology V. Gastroenterology. 61: 357-362. 